\title{
Modelos econométricos de estimativa da força de trabalho: uma revisão integrativa da literatura
}

I ${ }^{1}$ Cid Manso de Mello Vianna, ${ }^{2}$ Celia Regina Pierantoni, ${ }^{3}$ Tania Cristina França,
${ }^{4}$ Carinne Magnago, ${ }^{5}$ Marcus Paulo da Silva Rodrigues, ${ }^{6}$ Marina Campos Morici I

Resumo: A revisão integrativa da literatura objetivou levantar o estado atual do conhecimento sobre modelos econométricos de projeção da força de trabalho no Brasil e identificar o mais apropriado para aplicação no âmbito da Atenção Primária em Saúde. A busca se deu a partir de diversas fontes: bases de dados eletrônicas, revistas, anais de congressos, referência cruzada e Plataforma Lattes, por meio de descritores e palavras-chave. A amostra foi constituída de nove publicaçōes, as quais foram lidas, resumidas e analisadas. Os resultados apontam o uso de seis métodos de estimativa de recursos humanos distintos, porém em sua maioria as metodologias propõem cálculos preditivos para o dimensionamento de pessoal com vistas à alocação/ realocação imediata de profissionais. Ressaltase a impossibilidade de definir o melhor modelo econométrico para a projeção de recursos humanos em saúde na Atenção Primária, em virtude do pequeno número de publicações nacionais relativas ao tema, nas quais não se obteve homogeneidade, embora se verifique maior preocupação com a alocação da força de trabalho em saúde, mormente no âmbito hospitalar.

Palavras-chave: recursos humanos em saúde; força de trabalho; modelos econométricos; necessidades e demandas de serviços de saúde; técnicas de estimativa.
1 Professor adjunto do Instituto de Medicina Social, Universidade do Estado do Rio de Janeiro (IMS-UERJ), Rio de Janeiro, Brasil. Endereço eletrônico: cdvianna@gmail.com

2 Professora associada do IMS-UERJ, Rio de Janeiro, Brasil. Endereço eletrônico: cpierantoni@gmail.com

${ }^{3}$ Professora adjunta do IMS UERJ, Rio de Janeiro, Brasil. Endereço eletrônico: taniaf@ ims.uerj.br

${ }_{4}^{4}$ Pesquisadora da Estação de Trabalho IMS-UERJ da Rede de Observatório de Recursos Humanos em Saúde Rio de Janeiro, Brasil. Endereço eletrônico: carinne.mag@ gmail.com

${ }^{5}$ Pesquisador do Programa de Estudos em Avaliação de Tecnologias e Economia da Saúde, IMS-UERJ, Rio de Janeiro, Brasil. Endereço eletrônico:mps_rodrigues@ yahoo.com.br

${ }^{6}$ Pesquisadora visitante no IMS-UERJ, Rio de Janeiro, Brasil. Endereço eletrônico: marinamorici@gmail.com 
A Organização Mundial da Saúde (OMS), por meio do Relatório Mundial de 2006, chama a atenção para a crise nos recursos humanos em saúde (RHS), que vem impondo um impacto negativo sobre as capacidades de muitos países para promover e melhorar a saúde de suas populações. Assim, a adequada provisão de pessoal é apontada como uma estratégia fundamental para promoção e mudanças nos indicadores de saúde (OMS, 2007).

Essa posição é consubstanciada pela Agenda Nacional de Prioridades de Pesquisa em Saúde, que elegeu temas prioritários a serem pesquisados e que foram definidos através da interlocução entre gestores, pesquisadores e usuários. Dentre os temas, está a Gestão do Trabalho em Saúde, que destaca, entre seus principais tópicos, o desenvolvimento de metodologias para a projeção da força de trabalho (BRASIL, 2008).

O número de postos de trabalho em saúde no Brasil, sobretudo na Atenção Primária em Saúde (APS), foi ampliado em grande medida devido à expansão da rede de serviços de saúde após a implementação do Sistema Único de Saúde (SUS) (PIERANTONI et al., 2008). Consecutivamente, a Estratégia Saúde da Família (ESF), criada em 1994 pelo Ministério da Saúde (MS) com o objetivo de reorganizar os serviços e práticas sanitárias, passou a ser mais um campo nesse mercado de trabalho. Em 2001, estimava-se que pelo menos 170 mil postos de trabalho estavam envolvidos diretamente na condução do programa, sem contar o pessoal de apoio administrativo. No ano 2004, esse contigente já demonstrava um enorme incremento, chegando a contar com aproximadamente $400 \mathrm{mil}$ trabalhadores (NEY, 2009; MAGNAGO et al., 2011).

Embora tenha ocorrido a expansão de estruturas relacionadas à APS no país, para que a mesma se torne resolutiva e possa contemplar uma parcela significativa da população, é fundamental que haja planejamento do quantitativo da força de trabalho necessária à consecução dos objetivos do sistema nacional de saúde (ROSSELLI et al., 2001). Desta forma, o dimensionamento de RHS pode ser considerado a etapa inicial do processo de provimento de pessoal, e tem por objetivo antever o quantitativo e a composição de profissionais necessários ao desenvolvimento das atividades diárias de assistência. A partir do dimensionamento, é possível estimar os investimentos necessários à formação e 
capacitação de recursos humanos, bem como em infraestrutura e equipamentos para as unidades prestadoras de serviço.

A alocação de RHS, porém, não se restringe somente ao uso de instrumentos analíticos para quantificar indivíduos. É preciso calcular o número necessário de profissionais com competências específicas que as habilite a desempenhar tarefas consoantes aos princípios e necessidades dos serviços de saúde. Além disso, ela abarca o julgamento de potenciais mudanças do ambiente interno e externo, com destaque para transições demográficas e epidemiológicas, que podem, por sua vez, gerar diferentes demandas profissionais (BRASIL, 2006).

Modelos matemáticos mais complexos do que simples razões de profissionais por população ou setor parecem ser mais adequados ao cálculo da demanda por trabalhadores da saúde. Estes consideram diferentes variáveis, tais como: crescimento demográfico, padrão de adoecimento da população e relações entre mão de obra requerida e disponível, predizendo o equilíbrio mais adequado entre distribuição e número de trabalhadores de saúde. Embora as estimativas de demanda por profissionais de saúde sejam de fundamental importância no planejamento em saúde, o tema tem sido pouco discutido no âmbito da APS, como foi evidenciado em levantamento bibliográfico nacional e internacional sobre metodologias para o planejamento de RHS (PIERANTONI et al., 2011).

O referido estudo verificou que a maioria dos trabalhos encontrados aplicou cálculos para dimensionamento de pessoal para setores hospitalares especializados, como unidades de terapia intensiva (UTIs), com foco nos profissionais de enfermagem. Nesse cenário, o presente estudo descreve uma revisão integrativa da literatura, com a finalidade de conhecer os modelos de estimativa de força de trabalho utilizados no Brasil, e de subsidiar a escolha/desenvolvimento de um modelo a ser utilizado no âmbito da APS que considere as características do sistema de saúde brasileiro.

\section{Metodologia}

\section{Revisão integrativa}

A revisão integrativa da literatura científica é um método que permite compreender determinado fenômeno por meio da sumarização de múltiplos estudos científicos, subsidiando a tomada de decisão e incorporando evidências na prática profissional. É uma abordagem que permite a inclusão de estudos que 
adotam diversas metodologias. Esta revisão é operacionalizada por meio de seis etapas básicas, a saber: (1) formulação de questão de pesquisa; (2) estabelecimento de critérios de inclusão e exclusão; (3) coleta de dados; (4) avaliação dos estudos selecionados; (5) análise e interpretação dos resultados; e (6) apresentação da súmula do conhecimento (WHITTEMORE, 2005; MENDES; SIQUEIRA; GALVÃO, 2008; SOUZA et al., 2011).

Esta revisão integrativa objetivou responder à seguinte questão: "Qual o estado atual do conhecimento sobre o uso de modelos econométricos de estimativa da força de trabalho no Brasil?". Utilizou fontes abrangentes com diversas estratégias de busca sistematizadas, procedendo à seleção de publicações a partir de critérios predeterminados e que, em etapa posterior, sofreram apreciação crítica e compilação, tendo como produto uma súmula das evidências relacionadas ao tema de busca. Os critérios de inclusão estipulados foram: estudos nacionais independentes do desenho metodológico que abordaram como tema o uso de modelos para a previsão de demanda de recursos humanos, disponíveis na íntegra em meio online. Foram excluídos da pesquisa os artigos de reflexão, erratas e editoriais.

A pesquisa na literatura envolveu todas as bases de dados de literatura científica contidas na Biblioteca Virtual em Saúde (BVS) e na Perie, do Centro de Documentação do Instituto de Economia da Universidade Estadual de Campinas. Na tentativa de aumentar a abrangência da pesquisa, também se realizou busca diretamente em revistas científicas das áreas de administração, a saber: Revista de Administração da Universidade de São Paulo (RAUSP); Revista de Administração Pública (RAP); Revista de Administração de Empresas (RAE); Revista de Administração Contemporânea (RAC); Revista de Administração em Saúde (RAS); revista Planejamento e Políticas Públicas (PPP); revista Desafios do Desenvolvimento e; Boletim Mercado de Trabalho. Referências cruzadas dos artigos pré-selecionados e anais de eventos científicos também se constituíram como instrumentos de busca.

A coleta de dados foi realizada no período compreendido entre 27 de abril e 31 de maio de 2012, nas referidas fontes, por meio de descritores e palavras-chave. Os descritores utilizados foram selecionados na BVS através dos Descritores em Ciências da Saúde, edição 2012, quais sejam: "recursos humanos", "modelos econométricos" e "força de trabalho". Como forma de estender a sensibilidade da busca, selecionaram-se também as palavras-chave "previsão de pessoal", 
"trabalho", “turnover”, "rotatividade”, “capital humano”, “econometria”, "oferta de mão de obra" e "demanda por trabalho".

As revistas PPP, Desafios do Desenvolvimento, RAS e o Boletim Mercado de Trabalho foram consultadas por meio dos sumários de todas as edições disponíveis online. Adicionalmente, a fim de identificar grupos de pesquisa que realizam estudos sobre estimativas de recursos humanos e suas respectivas publicações não indexadas nas fontes pesquisadas e que, portanto, não poderiam ser encontradas nas etapas anteriores, foram realizadas buscas no Diretório dos Grupos de Pesquisa no Brasil, mecanismo de busca hospedado na Plataforma Lattes do Conselho Nacional de Desenvolvimento Cientifico e Tecnológico (CNPq). Foram considerados todos os grupos de pesquisa inseridos nas áreas da saúde e/ou ciências sociais aplicadas, independentemente da localização geográfica e vínculo institucional. Para compor a amostra final, foram selecionados os grupos com pesquisas relacionadas à estimativa de força de trabalho de qualquer categoria profissional e ambiente de trabalho, com o intuito de resgatar possíveis publicações não disponíveis nas bases de dados selecionadas.

O refino dos grupos de pesquisa foi realizado por meio da leitura dos títulos e objetivos das linhas de pesquisa. Após a pré-seleção, foram acessados os currículos dos líderes dos grupos em busca de produção bibliográfica de interesse para esta pesquisa.

\section{Seleção das publicações e análise}

A pré-seleção das publicações retornadas ocorreu em três etapas distintas. $\mathrm{Na}$ primeira, foi realizada pré-eleição das publicações por um grupo de três pesquisadores; refinos sucessivos mediante leitura dos títulos e resumos dos estudos foram necessários em virtude da alta sensibilidade alcançada, como consequência do uso dos diversos termos de busca. Na segunda etapa, as publicações préselecionadas foram revisadas separadamente por dois grupos, com três revisores cada. Cada grupo fez novos refinos, a fim de que a amostra final tivesse maior especificidade. Finalmente, os grupos realizaram reunião de consenso para a composição da amostra final, leitura completa e síntese dos trabalhos. Para tanto, um instrumento de análise foi elaborado, contendo as seguintes variáveis: título, periódico, ano de publicação, autoria, área de atuação dos pesquisadores, objetivos, metodologia de cálculo, sujeitos da pesquisa e resultados. 


\section{Resultados}

As buscas na BVS ( $\mathrm{n}=137)$, Perie $(\mathrm{n}=137)$, RAUSP $(\mathrm{n}=3)$, RAP $(\mathrm{n}=3)$, RAE $(\mathrm{n}=397)$ e RAC $(\mathrm{n}=19)$ retornaram 696 publicações, e após deliberação em reunião de consenso, apenas três compuseram a amostra final. Nas revistas Desafios do Desenvolvimento, PPP, RAS e Boletim Mercado de Trabalho, foram realizadas consultas ao sumário de todas as ediçôes online, a saber: 64 ( $\mathrm{n}=313$ artigos); 24 ( $\mathrm{n}=166$ artigos); 46 ( $\mathrm{n}=104$ artigos) e 35 ( $\mathrm{n}=35$ artigos), respectivamente, mas nenhum artigo foi selecionado. Por meio de referência cruzada e anais de congressos, selecionaram-se cinco publicações. A busca via Plataforma Lattes resultou na seleção de um estudo. A amostra final foi composta, portanto, de nove publicações, como mostra a Figura 1.

As variáveis identificadoras das publicações selecionadas encontram-se no quadro 1, mediante o qual se pode notar que cinco são artigos, dois são trabalhos apresentados em eventos científicos, um é relatório de pesquisa e outro é dissertação.

Observa-se, ainda, que $45 \%$ dos estudos foram publicados em periódicos de enfermagem, 22\% são da área de administração, estando os demais distribuídos igualmente nas áreas de logística (11\%), informática (11\%) e economia (11\%). Dois artigos foram publicados no mesmo periódico e apresentam um autor em comum. A distribuição por ano de publicação apontou que as publicações mais recentes são datadas de 2008.

Quanto aos objetivos propostos, identificou-se que todas as publicações abordam o dimensionamento de recursos humanos para área específica, com destaque para o setor saúde, com seis publicaçôes, o que representa pouco mais de $66 \%$ do total dos trabalhos selecionados. Ressalta-se, ainda, que destes seis, quatro tinham por sujeitos de pesquisa trabalhadores da categoria de enfermagem. Os demais trabalhos tiveram por foco a estimativa de pessoal em outros setores, quais sejam: indústria, tendo por população-alvo montadores e operadores do setor de fabricação de sanitários de uma empresa; telecomunicações, com projeção de recursos humanos para conformação de equipe de um Centro de Operações de Rede; e habitação, com previsão dos funcionários e estagiários de uma secretaria municipal. Incluem-se na amostra funcionários do setor de mão de obra, de elaboração e planejamento de projetos, entre outros. 


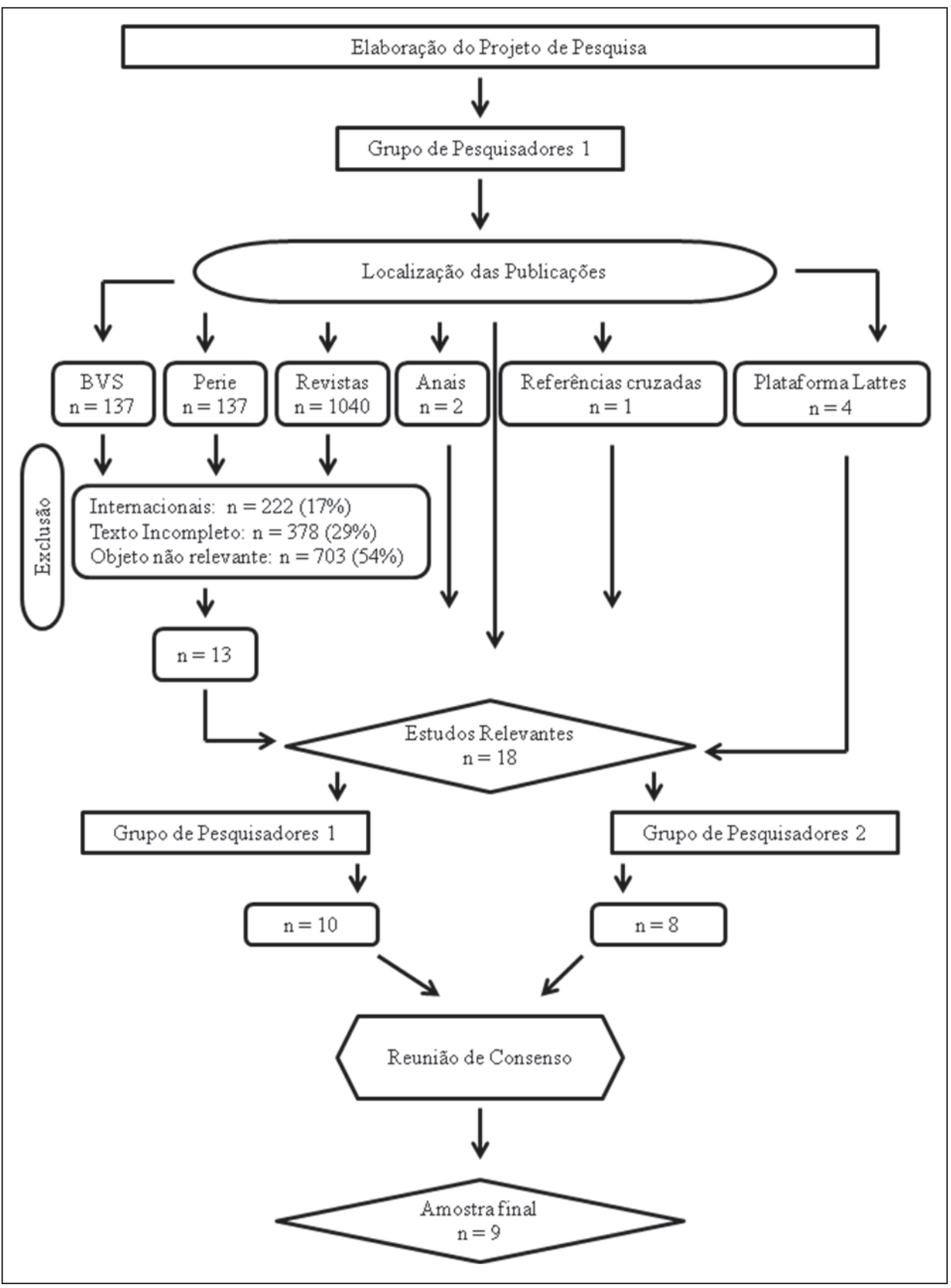




\begin{tabular}{|c|c|c|c|c|c|}
\hline$\frac{0}{0} \cdot \frac{0}{0}$ & 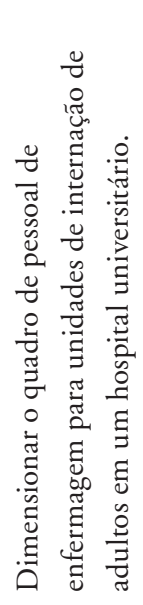 & 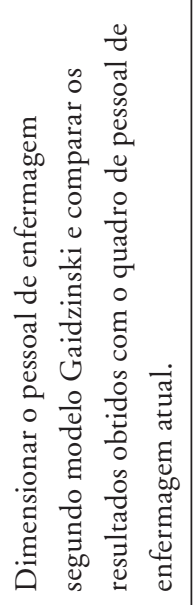 & 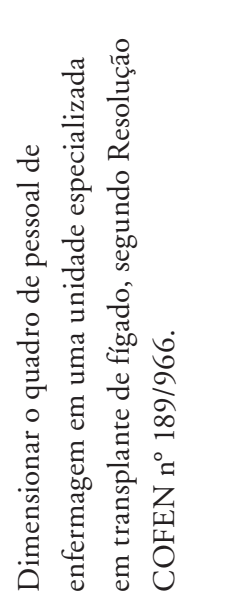 & 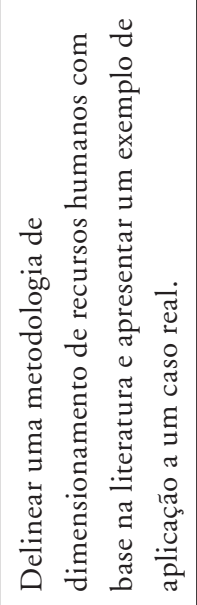 & 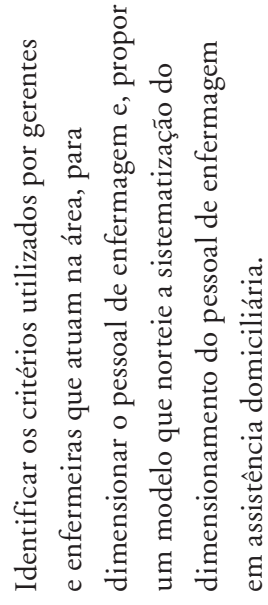 \\
\hline 氕 & 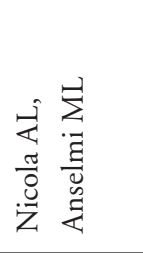 & 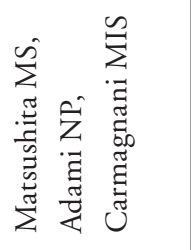 & 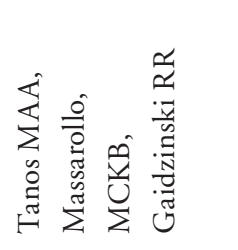 & 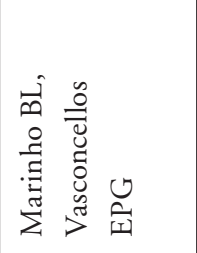 & 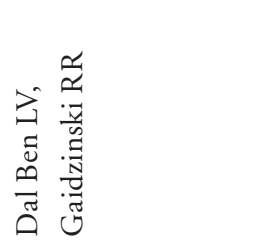 \\
\hline 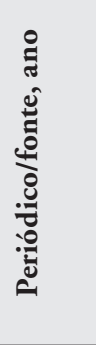 & 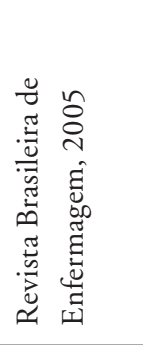 & 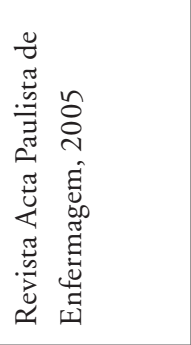 & 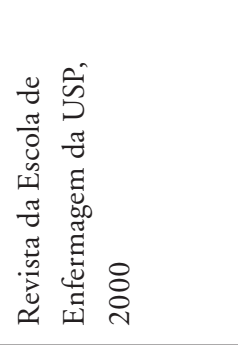 & 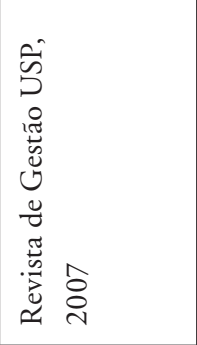 & 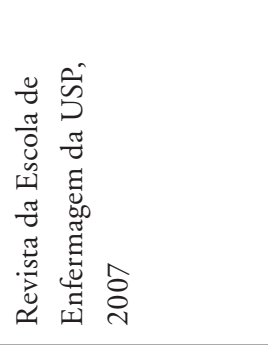 \\
\hline 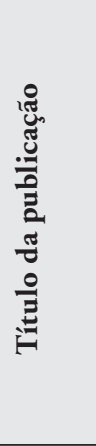 & 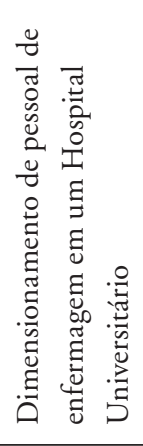 & 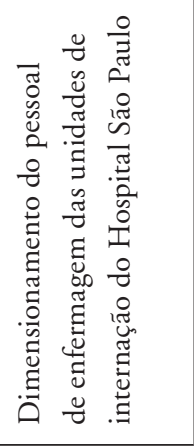 & 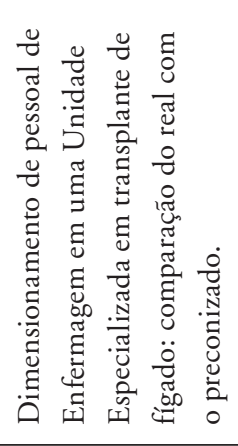 & 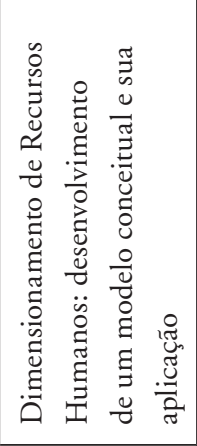 & 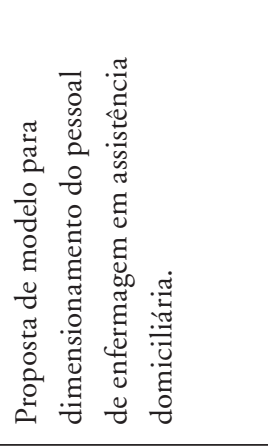 \\
\hline 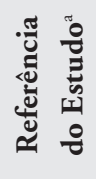 & $\Rightarrow$ & 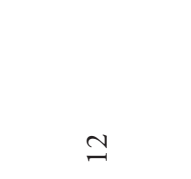 & 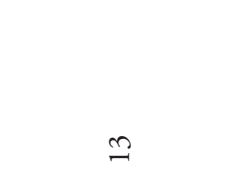 & $\simeq$ & $\bumpeq$ \\
\hline
\end{tabular}




\begin{tabular}{|c|c|c|c|c|}
\hline 苞 & 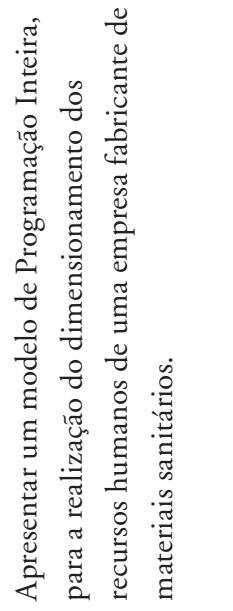 & 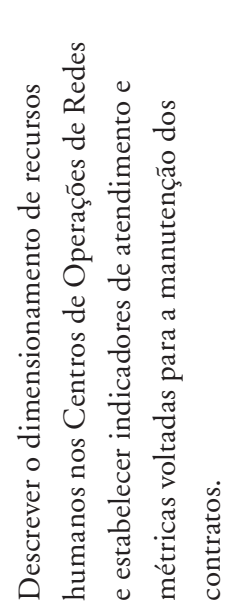 & 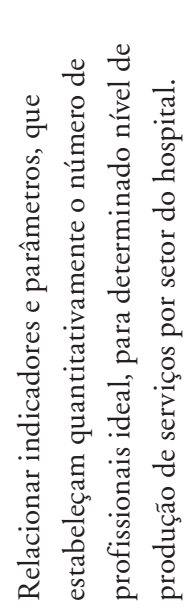 & 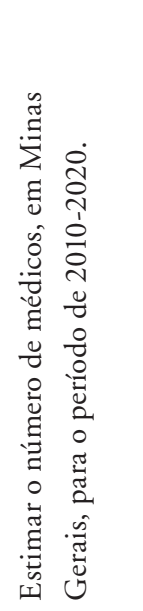 \\
\hline 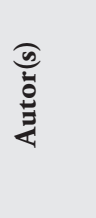 & 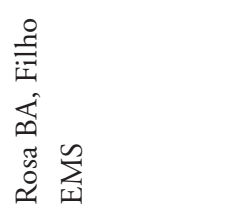 & 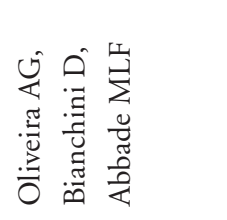 & 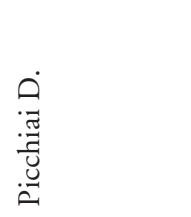 & 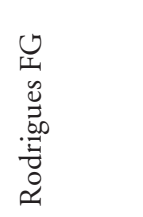 \\
\hline 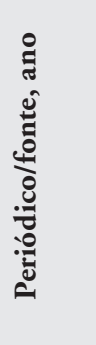 & 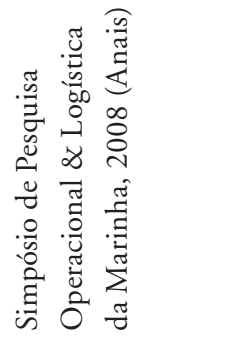 & 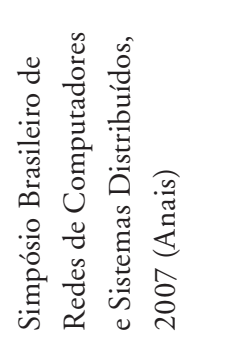 & 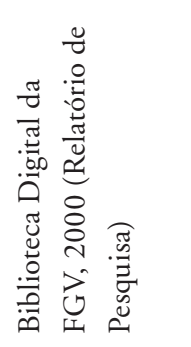 & 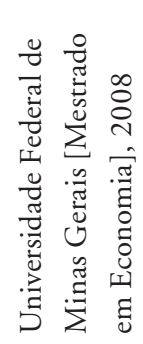 \\
\hline 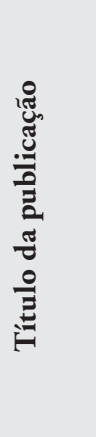 & 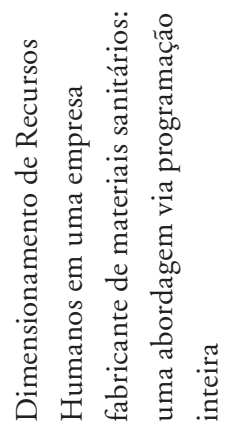 & 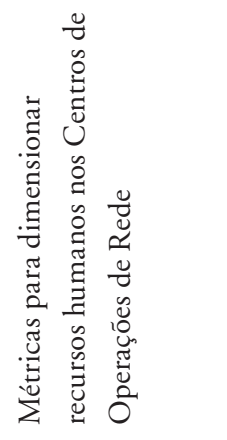 & 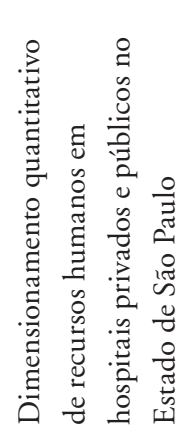 & 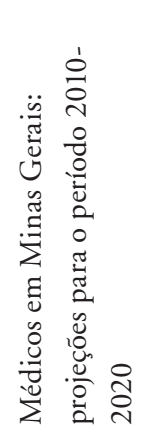 \\
\hline 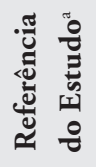 & $\stackrel{\ddots}{\circ}$ & $\beth$ & $\stackrel{\infty}{\sim}$ & 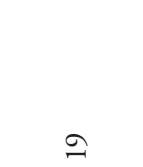 \\
\hline
\end{tabular}


Exceto pelo estudo de Picchiai (2000), que objetivou relacionar indicadores e parâmetros para estabelecer quantitativo de pessoal, os demais apresentam a aplicação de métodos de previsão de recursos humanos. Não se constatou, portanto, grande diversidade entre os objetivos identificados. No que se refere ao desenho metodológico, o estudo de caso foi o mais utilizado, aparecendo em cinco dos nove trabalhos, seguido do estudo descritivo, empregado em três publicações e, finalmente, da revisão de literatura, que foi aplicada em apenas um trabalho, como elucidado pelo quadro 2 .

Quadro 2. Distribuição das publicações segundo tipo de estudo e métodos de previsão de recursos humanos empregados, Brasil, 2012

\begin{tabular}{|l|l|l|}
\hline Estudo & \multicolumn{1}{|c|}{ Tipo de estudo } & \multicolumn{1}{c|}{ Método de previsão de recursos humanos } \\
\hline 11 & Estudo de caso & Método Gaidzinski \\
\hline 12 & Descritivo & Método Gaidzinski \\
\hline 13 & Estudo de caso & Método Gaidzinski \\
\hline 14 & Estudo de caso & Método proposto pelos autores \\
\hline 15 & Descritivo & Método Gaidzinski \\
\hline 16 & Estudo de caso ${ }^{\mathrm{a}}$ & Modelo de Programação Inteira (PI) \\
\hline 17 & Estudo de caso $^{\mathrm{a}}$ & Modelo da Teoria de Filas \\
\hline 18 & Revisão de literatura $^{\text {Modelo de Hospitais Universitários }}{ }^{\mathrm{b}}$ \\
\hline 19 & Descritivo $^{\mathrm{a}}$ & $\begin{array}{l}\text { Método dos Componentes Demográficos e Método } \\
\text { proposto por Goic }\end{array}$ \\
\hline
\end{tabular}

${ }^{a} \mathrm{O}$ desenho de estudo não foi classificado pelos autores; portanto, a classificação foi realizada a partir das características e descrição dos passos metodológicos.

${ }^{\mathrm{b}} \mathrm{O}$ estudo não aplica o modelo, mas apresenta-o e o descreve.

Seis metodologias distintas de estimativa/projeção de recursos humanos empregados no Brasil foram identificadas nas publicações selecionadas.

\section{Método Gaidzinski}

O método proposto por Gaidzinski (1998) foi formulado com vistas a subsidiar o dimensionamento dos profissionais de enfermagem para instituições hospitalares a partir de cinco diferentes etapas: (a) conhecimento do perfil dos 
pacientes quanto ao grau de complexidade assistencial; (b) levantamento da necessidade de horas de assistência de enfermagem de acordo com a Resolução do Conselho Federal de Enfermagem (COFEN) no 189/96; (c) determinação do percentual dos diferentes profissionais de enfermagem demandados a partir da resolução mencionada, que estabelece a distribuição percentual de profissionais de enfermagem com base no Sistema de Classificação de Pacientes. Este método busca agrupar os pacientes segundo o nível de dependência assistencial; (d) identificação da jornada efetiva de trabalho, uma vez que se pressupõe que os trabalhadores não são produtivos em todo o turno de trabalho, pois dispensam tempo em atividades não relacionadas às profissionais, tais como descanso, atendimento das necessidades fisiológicas e outras; e (e) identificação do percentual de absenteísmo e ausências da equipe de enfermagem.

Seguidas essas etapas, aplica-se a seguinte equação para calcular o quantitativo de profissionais da categoria de enfermagem necessário aos cuidados exigidos pela unidade:

$$
q=\sum_{k} \frac{P_{k}}{100} \cdot \frac{\sum_{j}\left(n_{j} \cdot h_{j}\right)}{t \cdot p} \cdot\left[\left(1+\frac{e}{d-e}\right) \cdot\left(1+\frac{f}{D-f}\right) \cdot\left(1+\frac{v_{k}}{D-v_{k}}\right) \cdot\left(1+\frac{a_{k}}{D-a_{k}}\right)\right]
$$

\section{Onde:}

$\mathrm{q}$ = quantidade total de pessoas de enfermagem;

$\mathrm{Pk}=$ proporção percentual da categoria $\mathrm{k}$;

$\mathrm{k}=$ categoria profissional;

nj = quantidade média diária de pacientes que necessitam do tipo de cuidado j;

$\mathrm{j}$ = tipo de cuidado requerido pelo paciente (mínimo, intermediário, semi-intensivo, intensivo);

hj = tempo médio de cuidado de cada paciente que necessita do cuidado j;

$\mathrm{t}$ = tempo diário de trabalho de cada profissional dado em horas;

$\mathrm{p}=$ produtividade média ( $\%$ do tempo diário de trabalho, dedicado ao cuidado do paciente);

e = número de folgas semanais que necessitam de cobertura;

$\mathrm{f}=$ número de dias de feriados por ano, não coincidentes com o domingo;

$\mathrm{vk}=$ número de dias de férias por ano, por categoria profissional;

$\mathrm{ak}$ = número médio de dias de ausências não previstas (absenteísmo) por categoria profissional, por ano;

$\mathrm{d}=$ dias de funcionamento da unidade;

$\mathrm{D}=$ dias trabalhados no ano. 
Quatro estudos selecionados abordam o modelo de cálculo proposto por Gaidzinski. Três deles comparam o quadro existente com o preconizado (ou ideal). Os resultados obtidos por Nicola e Anselmi (2005) e Matsushita, Adami e Carmagnani (2005) apontaram quantitativo de enfermeiros existente nas unidades inferior ao projetado, e número de técnicos de enfermagem que ultrapassou o estimado. O estudo de Tanos, Massarollo e Gaidzinski (2000) assinalou um quadro de profissionais de enfermagem superior ao preconizado pela Resolução COFEN no 189/96. Ressalta-se que este último adotou os índices de absenteísmo fundamentados nos estudos de Gaidzinski et al. (1998), por considerar elevados aqueles encontrados por meio de observação e coleta de dados na própria unidade. Os índices estabelecidos pela literatura podem não corresponder à realidade de diversas unidades, portanto sua generalização e consequente utilização em detrimento das especificidades de cada local de trabalho podem gerar resultados não fidedignos.

O estudo de Dal Ben e Gaidzinski (2007), por sua vez, adaptou o método de modo que ele pudesse ser utilizado para calcular a necessidade de profissionais de enfermagem no atendimento domiciliar. Para tanto, fez-se necessário conhecer as atividades de cuidado exercidas pela enfermagem no domicílio. As autoras obtiveram essa informação mediante entrevista com profissionais relacionados à assistência domiciliária. Ressalta-se, entretanto, que ainda constitui um desafio estimar o tempo gasto nas atividades desenvolvidas no âmbito domiciliar, em virtude dos diversos graus de complexidade da assistência realizados, uma vez que são realizados atendimentos de pacientes com problemas diversos de saúde que podem requerer assistência de enfermagem integral, parcial ou apenas auxílio na realização de atividades de autocuidado; e pelo atendimento multiprofissional característico da assistência domiciliária. Esses fatores podem engendrar um cálculo não legítimo da média de horas gastas com as intervenções realizadas especificamente pelos profissionais de enfermagem.

\section{O Método da Teoria de Filas}

O método da Teoria de Filas baseia-se na probabilidade de formação de filas e, por meio de análises matemáticas, permite o dimensionamento da oferta de profissionais, com o propósito de atender às necessidades da clientela, mesmo num 
sistema de serviços no qual a demanda cresce de forma imprevisível. Este método considera a taxa de utilização dos serviços, a dinâmica do atendimento ao cliente e o tempo de permanência na fila. A taxa de utilização do sistema $(\rho)$ é dada por:

$$
\rho=\frac{\lambda}{c \beta}
$$

Onde:

$\lambda=$ ritmo médio de chegada de usuário no sistema;

$c=$ quantidade de profissionais;

$\beta=$ ritmo médio de atendimento no sistema.

A quantidade média de clientes no sistema $(n)$ é expressa através de:

$$
n=\frac{c \cdot \rho+\rho \cdot P q}{1-\rho}
$$

Onde:

$P q=$ probabilidade de os analistas estarem ocupados.

A probabilidade de ocupação da fila é indicada por:

$$
P q=\frac{(c \cdot p)^{c} \cdot P_{0}}{c !(1-\rho)}
$$

A probabilidade de o sistema estar vazio, ou seja, sem clientes $\left(P_{0}\right)$, é dada por:

$$
P_{0}=\sum_{n=0}^{c-1}\left[\frac{(c . \rho)^{n}}{n !} \pm \frac{(c . \rho)^{c}}{c !(1-\rho)}\right]^{-1}
$$

Finalmente, o tempo médio de permanência dos clientes na fila $(T F)$ é expresso por:

$$
T F=\frac{\left(\frac{\lambda}{\beta}\right)^{c}}{\beta \cdot c(1-\rho)^{2} c !}
$$


A aplicação deste método por Oliveira, Bianchini e Abbade (2007) se deu em uma empresa de telecomunicações, a partir de dados históricos do datacenter de uma operadora; os resultados da teoria de filas, comparados aos de simulações com distribuição estatística, não demonstraram correlação direta entre $T F$ e $\rho$ de $100 \%$ e o comportamento de $\lambda$ e $\beta$. Em virtude deste achado, os autores adaptaram o modelo, agregando uma variável de produtividade em dependência do tempo denominada $\beta \max$, que é calculada pela quantidade máxima de atendimentos por hora $\left(\beta_{h}=3\right.$ por analista/hora/mês) com o número de horas trabalhadas no mês ( $q H H=180$ horas), de forma que a quantidade máxima de atendimentos mensais por analista é:

$$
\beta_{h} m e \hat{e}=\beta_{h} \cdot q H H,
$$

que, adequado ao número da equipe, se verifica pela fórmula

$$
\beta_{\max }=\beta_{h} \text { mês.c }
$$

Após a reprodução dos dados utilizados nas simulações e aplicação da variável $\beta \max$, constatou-se que o dimensionamento adequado foi alcançado, uma vez que indicaram o real comportamento dos atendimentos e da taxa de ocupação.

\section{Modelo de Programação Inteira}

O modelo de Programação Inteira para dimensionamento de pessoal busca a utilização ótima dos recursos disponíveis, tendo em vista as limitações impostas pelo processo produtivo. Requer a definição de parâmetros de produtividade e da taxa de utilização dos serviços. Para tanto, faz-se necessário conhecer o tempo gasto na execução de cada tarefa realizada pelo profissional.

Rosa e Filho (2008) desenvolveram um modelo de programação inteira cuja função objetivo minimiza o custo total de alocação nas tarefas, dado um número fixo de profissionais que possuem a mesma produtividade unitária:

$$
\text { Minimizarz }=\sum c_{i j} x_{i_{j}}
$$

Adicionalmente, os autores acrescentaram duas restrições. A restrição 1 indica que o tempo total gasto pelos trabalhadores na realização de suas tarefas não pode exceder sua jornada de trabalho. A restrição 2 indica que determinada 
tarefa só poderá ser alocada a um profissional. As restrições são representadas pelas seguintes equações:

$$
\sum_{i} w_{i j} x i_{j} \leq b \quad \sum_{j} x_{i j}=1 \quad x i j \in\{0,1\}
$$

\section{Onde:}

$x_{i j}$ : variável de decisão que indica se o i-ésimo profissional é alocado na tarefa j;

$b$ : número máximo de horas que um profissional pode trabalhar;

$W_{i j}$ : tempo de realização da tarefa j pelo profissional i-ésimo montador;

$c_{i j}$ : custo de realização da tarefa j pelo profissional i-ésimo montador.

O modelo se mostrou útil no tocante a seu propósito de dimensionar pessoal com tarefas invariáveis e em pequeno número.

\section{Método das Componentes Demográficas e Método Goic}

O método das Componentes Demográficas, aplicado por Rodrigues (2008), consiste em projetar o número de profissionais de determinada faixa etária para o quinquênio seguinte, e a partir deste para o próximo quinquênio, e assim sucessivamente. Destaca-se que os componentes demográficos (fecundidade, mortalidade e migração) são as principais variáveis deste método. As projeções estão diretamente relacionadas às conjecturas do comportamento futuro dessas variáveis (GIVISIEZ, 2004).

O método Goic foi adaptado e também utilizado por Rodrigues (2008). Esse método é semelhante ao das Componentes Demográficas, uma vez que também estima a população e as variáveis responsáveis pelo comportamento de entrada e saída de médicos no mercado de trabalho, tais como migração e mortalidade.

O cálculo de projeção aplicado por Rodrigues (2008), com fundamento nos dois métodos referidos anteriormente, parte do estoque de médicos ativos de um ano base. Dado esse estoque, seguem-se os seguintes passos:

Passo 1: determinar a população de profissionais sobreviventes (fechada à migração), que corresponde ao número de profissionais ativos no momento $t$, ou seja, o estoque de profissionais ativos sobreviventes no final de um período de cinco anos, que é obtido por:

$$
{ }_{5} P_{x+5, F}^{t+5}={ }_{5} P_{x, s}^{t} *{ }_{5} S_{x, 5}
$$


${ }_{5} P_{x+5, F}^{t+5}=$ população fechada $(\mathrm{F})$ de profissionais ativos, por sexo, que passados cinco anos após $\mathrm{o}$ ano $t$, tinha $\mathrm{x}+5 \mathrm{a} \mathrm{x}+10$ anos;

${ }_{5} P_{x, s}^{t}=$ população de profissionais ativos, por sexo, que no ano $t$ tinha de $\mathrm{x}$ a $\mathrm{x}+5$ anos;

${ }_{5} S_{x, 5}=$ razão de sobrevivência por sexo, e por período quinquenal, ou seja, a probabilidade de um profissional ativo com $\mathrm{x} \mathrm{a} \mathrm{x}+5$ anos, no ano $t$, sobreviver ao final dos próximos cinco anos.

Passo 2: Determinar as novas entradas de profissionais ativos no período quinquenal que precede o ano da projeção, que depende do quantitativo de vagas ofertadas e ocupadas para o curso de graduação profissional no ano $t$, e que resultam em egressos no ano $\boldsymbol{t}+\boldsymbol{n}$ (n representa o período de duração do curso profissional). Desses egressos, um percentual se inscreverá nos conselhos profissionais:

$$
{ }_{5} P_{0, F}=N_{\Delta t} * S_{b}
$$

\section{Onde:}

$N_{\Delta t}=$ novos registros nos conselhos profissionais;

$S_{b}=$ razão de sobrevivência.

Passo 3: Determinar a população de profissionais aberta à migração que corresponde ao total projetado da população fechada, adicionada ao saldo migratório (SM) que ocorrera cinco anos antes dessa projeção:

$$
{ }_{5} S M_{x}^{t, t+5} \text { sexo }={ }_{5} I_{x}^{t, t+5} \text { sexo }-{ }_{5} E_{x}^{t, t+5} \text { sexo }
$$

Onde:

${ }_{5} I_{x}^{t, t+5}{ }_{\text {sexo }}=$ profissionais imigrantes, dados pelos egressos que se inscreveram em outro local (município, estado) entre $\boldsymbol{t}$ e $\boldsymbol{t}+5$ por sexo;

${ }_{5} E_{x}^{t, t+5}{ }_{\text {sexo }}=$ profissionais emigrantes, dados pelas transferências de profissionais para outro local (município, estado).

Passo 4: Determinar a taxa líquida migratória (TLM) obtida por:

$$
{ }_{5} T L M_{x_{\text {sexo }} 5}^{t} S M^{t}{ }_{\text {sexo }} /{ }_{5} P_{x \text { sexo }}^{t}
$$

Onde:

${ }_{5} P_{x}^{t}=$ estoque de profissionais por grupo etário e sexo. 
A análise das componentes utilizadas por este modelo permitiu esboçar um cenário de evolução futura do volume de médicos por sexo e por idade no Estado de Minas Gerais.

\section{Modelo proposto por Marinho e Vasconcelos}

Os autores (MARINHO; VASCONCELOS, 2007) apresentaram uma metodologia conceitual de dimensionamento de recursos humanos baseada na literatura e descreveram sua aplicação em uma empresa estatal. Para aplicação do método, levantaram-se: o cenário atual da organização no que se refere à carga de trabalho para cada atividade, perfil dos trabalhadores e padrões internos de desempenho (metas); evolução do quadro funcional e da produção a partir de dados históricos; e benchmarking. A confluência dessas informaçōes resultou no quadro ideal de recursos humanos a ser contratado.

Inicialmente, obteve-se o levantamento do quadro atual de profissionais mediante cálculo das horas ocupadas por mês por um trabalhador $\left(\mathrm{H}_{m}\right)$, a partir da equação 1. Estimou-se, em seguida, o levantamento do quadro necessário, calculado a partir das horas necessárias por mês por um trabalhador $\left(\mathrm{H}_{n}\right)$, obtidas com base na expectativa de evolução na produção e que, somadas à produção atual, resultaram na meta diária a ser alcançada por cada trabalhador $(m)$. Ademais, utilizou-se um índice de horas trabalhadas $\left(i_{h}\right)$, produto da subtração do total de horas trabalhadas pelo total de horas ociosas em virtude de férias, licenças, deslocamentos dentro da unidade, telefonemas, atendimento das necessidades fisiológicas e outros, como evidenciado pela equação 2.

$$
H_{m}=\left(\frac{d . p}{60}\right) . n \text { (1) } \quad H_{n}=\frac{\left(\frac{m \cdot d}{60}\right)}{i_{h}} . n
$$

Onde:

$d=$ duração da tarefa em minutos;

$p=$ produção por dia em quantidades;

$n=$ total de dias trabalhados no mês.

Segundo os autores, a aplicação dessa metodologia comprovou seu potencial, gerando resultados satisfatórios para a empresa, muito em parte devido à utilização de diferentes fontes e, sobretudo, por considerar a opinião daqueles que conhecem a organização. 


\section{Modelos hospitalares}

O estudo de Picchiai (2000) discorre sobre alguns modelos de cálculo de pessoal, embora objetive apenas relacionar os parâmetros e indicadores com as variáveis condicionantes do dimensionamento quantitativo de recursos humanos em hospitais. Grande parte dos parâmetros e indicadores levantados por esse estudo está baseada no modelo de Hospitais Universitários (BRASIL, 1992) e suas adaptações, que em linhas gerais trabalha fortemente a relação de funcionários por leito e se baseia na seguinte equação:

$$
P_{n}=\frac{\left(L \cdot t_{x}\right) \cdot h_{j}}{J_{s}}+I S T
$$

Onde:

$P_{n}=$ quantitativo de pessoal necessário;

$L=$ numero de leitos;

$t_{x}=$ taxa de ocupação;

$h_{l}=$ horas exigidas por profissional por leito;

IST = índice de segurança técnico (para suprimento de faltas, férias, etc.);

$J_{s}=$ jornada de trabalho semanal.

Os modelos hospitalares, embora sejam de natureza normativa, permitem dimensionar o número de profissionais de saúde e apoio administrativo de qualquer unidade hospitalar, visto que se baseiam em parâmetros definidos segundo a categoria profissional e o setor de trabalho.

\section{Modelos preditivos e a Atenção Primária em Saúde}

A grande dificuldade em planejar a força de trabalho reside na necessidade de prever um quantitativo suficiente de profissionais para atender a uma demanda de perfil elástico a qual não se tem certeza que existirá, sobretudo quando falamos de um sistema de saúde universal, não obstante a escassez da oferta e a baixa qualidade dos serviços disponíveis (PATTEN, 1971; MARINHO; VASCONCELOS, 2007; OCKÉ-REIS; ANDREAZZI; SILVEIRA, 2006).

Com referência aos métodos evidenciados nesta pesquisa, nota-se que, para a aplicação do método Gaidzinski, a demanda efetiva de atendimento da unidade deve ser conhecida detalhadamente. Além disso, utiliza-se como parâmetro 
de base a demanda atual, e não uma projeção, pressupondo, portanto, que as necessidades de saúde se manterão constantes ao longo do tempo. E uma vez que o método não considera a oferta de profissionais no mercado de trabalho, implica assumir que o estoque na unidade de análise é invariável. Além disso, quando for necessário contratar profissionais para complemento de quadro, haverá estoque compatível no mercado e os salários oferecidos serão suficientes para atração de novos profissionais. O método Gaidzinski pode ser útil para a projeção de profissionais de enfermagem para atuar em unidades hospitalares, onde é possível determinar o tempo despendido em assistência de enfermagem com pacientes internados, considerando seu grau de complexidade. A aplicação deste método para outros profissionais de saúde, bem como no âmbito da APS, requer grandes adaptações e não garante que uma meta estimada de RHS seja alcançável.

Os modelos baseados na Teoria das Filas têm sido largamente empregados em saúde para cálculo do tempo de espera de pacientes nas filas de atendimento. Por exemplo, Marinho (2006) calculou os tempos nas filas para transplantes, e Marinho e Cardoso (2007) avaliaram os tempos de espera no SUS para internações relacionadas à gravidez, parto e puerpério. Embora relativamente simples, sua aplicação no campo da saúde para os fins descritos é de difícil aplicação, sobretudo na APS, em virtude de sua complexidade operacional. Para tanto, isso requer o conhecimento dos intervalos de tempo entre as chegadas dos pacientes nas unidades (para cálculo da demanda espontânea) e o início e término dos diferentes atendimentos executados pelos profissionais (cálculo do tempo gasto com as atividades). Ademais, os modelos de Teorias das Filas não contemplam variáveis relacionadas à adequação da distribuição dos serviços, nem mudanças nas taxas de utilização desses mesmos serviços.

O modelo de programação inteira, por sua vez, pressupõe que não haja interferências externas ao trabalho, que a demanda de serviços seja previamente determinada e que todos os trabalhadores possuam a mesma capacidade produtiva. Desconsidera a dinamicidade e os imprevistos no ambiente de trabalho, bem como as mudanças das necessidades da clientela (ROSA; FILHO, 2008; BARBER; LÓPEZ-VALCÁRCEL, 2010). Pode ser aplicado para a alocação diária de recursos humanos, especialmente em setores nos quais a demanda por serviços permaneça constante e a diversidade das atividades realizadas seja pequena. Sua aplicação direta na APS talvez não seja possível, pois implicaria desconsiderar a 
demanda espontânea, assumir que existe um padrão de produtividade predefinido para o setor saúde, e que todas as atividades desenvolvidas e o tempo despendido para cada uma delas fossem homogêneos entre os profissionais.

O método adotado por Rodrigues (2008), que é uma associação entre o método das componentes demográficas e o método Goic, considera o crescimento obtido nos anos anteriores para calcular o desenvolvimento das variáveis para os anos seguintes, visto que pressupõe que a taxa de crescimento e as características demográficas variam previsivelmente. Não considera alteraçôes nas necessidades de atendimento da população nem a distribuição dos serviços de saúde. Ele se atém a projetar o número de profissionais que estarão disponíveis no mercado (estoque), considerando apenas o número de habitantes e, portanto, também não considera a funcionalidade e a alocação desses profissionais. Apesar do exposto, trata-se de um método economicamente viável e mais bem aplicável quando a finalidade é alterar os padróes de oferta dos serviços de saúde (AHMED; FATEHA; BENJAMIN, 2000; HALL; MEJIA, 1978).

A metodologia utilizada por Marinho e Vasconcelos (2000) deixou algumas lacunas: não esclarece a influência do benchmarking; não discute a interferência dos dados históricos nem das políticas governamentais para o setor; e não contempla índices de rotatividade de profissionais. Por ter sido aplicado em uma empresa estatal, onde esse índice é mínimo - em virtude do modelo de contratação estatutário vigente - não se podem garantir resultados de projeção fidedignos caso seja aplicada na APS, uma vez que a taxa de rotatividade de profissionais atuantes nos serviços básicos de saúde é extremamente alta (CAMPOS; MALIK, 2008; MEDEIROS et al., 2010). Embora o modelo conceitual esteja bem descrito, sua aplicação não ficou explícita em virtude da sumarização das etapas, do não acesso aos instrumentos de coleta de dados e de equívocos na apresentação e descrição de equações, deixando dúvidas no que se refere aos métodos de cálculo empregados, sobretudo na fase de ajustes.

O modelo hospitalar, embora facilmente inteligível e aplicável, se torna frágil na medida em que se fundamenta basicamente na carga horária de trabalho por leito/paciente internado, inviabilizando a aplicação em outros cenários de saúde. Além disso, condiciona a aplicação do modelo ao uso de diversos parâmetros predefinidos, como: tipo de clientela e dependência dos serviços; condições de trabalho oferecidas aos funcionários; nível de complexidade dos serviços oferecidos; 
grau de resolutividade e de tecnologia incorporada. Contudo, cabe delinear que o estudo aplicado por Picchiai (2000) pode contribuir de forma significativa para a escolha e adaptações de variáveis a serem empregadas no âmbito da APS.

Em linhas gerais, os métodos empregados nas publicações selecionadas centram-se no dimensionamento do quadro de pessoal, cuja estimativa é realizada normalmente com base na demanda por serviços, existente ou estimada, e tem como uma das principais variáveis o tempo gasto com as atividades, o que concede a esses modelos uma lógica taylorista/fordista. Esses modelos não consideram possíveis imprevistos externos à organização, pois propõem cálculos para o dimensionamento de pessoal com vistas à alocação/realocação imediata de profissionais. A exceção é o modelo utilizado por Rodrigues (2008), que trabalha um modelo de projeção com vistas ao conhecimento do número de profissionais existentes no futuro.

Em que pese uma possível adaptação dos modelos econométricos de dimensionamento de recursos humanos para aplicação na APS, algumas limitações se impõem: (a) os modelos são utilizados para aplicação em setores fechados, onde há uma gama de funções específicas e similares e um número estabelecido de leitos; (b) o cálculo utiliza como principal variável o tempo real dispensado em cada procedimento/função ou em parâmetros de tempo normatizados pela organização, ou seja, a previsão de pessoal é realizada com base na carga de trabalho.

Um modelo de projeção a ser aplicado na APS deve agregar diversas variáveis temporais, tais como: as socioeconômicas (IDH, renda); epidemiológicas e demográficas (mortalidade, migrações, envelhecimento) uma vez que elas implicam mudanças salariais, demandas de serviços de saúde e quantitativo populacional, respectivamente.

Ademais, outro indicador fundamental é o índice de rotatividade de recursos humanos ou turnover, que geralmente é produto da insatisfação dos trabalhadores com o contexto do trabalho e que, por sua vez, pode estar relacionada a diversos fatores identificáveis, como baixos salários, péssimas condições estruturais e carga de trabalho excessiva (NEY, 2009; VARELLA; PIERANTONI, 2008, PIERANTONI et al., 2010; MAGNAGO, 2012).

A análise dos estudos aponta que o modelo adotado por Rodrigues apresenta maior possibilidade de ser aplicado na APS, pois é constituído por um modelo de projeção que considera variáveis importantes para o contexto da saúde. 
Entretanto, dentro do pequeno número de trabalhos encontrados por esta revisão integrativa não houve homogeneidade e, por consequência, não se pode inferir uma consensualidade sobre os modelos de estimativa mais apropriados para aplicação na área da saúde.

\section{Conclusões}

Evidencia-se a impossibilidade de definir, a partir da revisão integrativa da literatura, qual o melhor modelo para a estimativa de força de trabalho para a APS em virtude do pequeno número de publicações nacionais relativas ao tema. Não há, portanto, subsídios suficientes que sustentem a escolha de determinada metodologia.

O emprego de diferentes métodos para diferentes categorias profissionais e ambientes, cada um com suas limitações e vantagens, permite identificar inúmeras variáveis a serem relevadas no momento da construção/aplicação de um método de estimativa de recursos humanos em saúde, tais como produtividade, rotatividade, tipo de serviço, determinantes demográficos, entre outros.

Embora o tema seja escasso na literatura científica nacional, não obstante o aumento expressivo da produção científica brasileira, visualiza-se, nas publicações existentes, maior preocupação com a alocação de RHS, sobretudo no âmbito hospitalar. Destarte, urge a necessidade de pesquisas mais abrangentes, enlaçando o contexto internacional, a fim de identificar outras evidências que possam corroborar as encontradas por esta revisão e, portanto, subsidiar a tomada de decisão frente a diferentes modelos de estimativa de pessoal e em virtude das especificidades do setor saúde, especialmente dos serviços de atenção primária. ${ }^{1}$

\section{Referências}

AHMED, A.A.; FATEHA, B.; BENJAMIN, S. Demand and supply of doctors and dentists in Bahrain, 1998-2005. Eastern Mediterranean Health Journal, v.6, n.1, p.6-12, 2000.

BARBER, P.; LÓPEZ-VALCÁRCEL, B.G. Forecasting the need for medical specialists in Spain: application of a system dynamics model. Human Resources for Health, v.8, n.24, p.1-9, 2010.

BRASIL. Ministério da Educação. Programa de Estudos Avançados em Administração Hospitalar e de Sistemas de Saúde do Hospital das Clínicas da Faculdade de Medicina da Universidade de São Paulo e da Fundação Getúlio Vargas. Modelo de dimensionamento de recursos humanos para hospitais universitários. São Paulo, 1992. 
Europeia. Textos de referência em gestão de pessoas: dimensionamento de força de trabalho. Brasília, 2006. 76p.

Ministério da Saúde. Secretaria de Ciência, Tecnologia e Insumos Estratégicos. Departamento de Ciência e Tecnologia. Agenda nacional de prioridades de pesquisa em saúde. 2. ed. Brasília : Editora do Ministério da Saúde, 2008.

CAMPOS, C.V.A.; MALIK, A.M. Satisfação no trabalho e rotatividade dos médicos do Programa de Saúde da Família. Revista de Administração Pública, v.42, n.2, p.347-68, 2008. CONSELHO FEDERAL DE ENFERMAGEM. Resolução n 189/96. Estabelece parâmetros para dimensionamento do quadro de profissionais de enfermagem nas instituições de saúde. In: Documentos básicos de enfermagem: enfermeiros, técnicos e auxiliares. São Paulo, 1997. p.177-80.

DAL BEN, L.W.; GAIDZINSKI, R.R. Proposta de modelo para dimensionamento do pessoal de enfermagem em assistência domiciliária. Revista Escola de Enfermagem USP, v.41, n.1., p.97-103, 2007.

GAIDZINSKI, R.R. et al. Estudo das ausências da equipe de enfermagem num hospital geral de grande porte. Revista Enfermagem Complexo HC/FMUSP, v.1, n.4, p.8-14, 1998.

GIVISIEZ, G.H.N. Introdução a métodos de estimativas e interpolação populacionais. In: RIANI, J.L.R.; RIOS-NETO, E.L.G. (Org.). Introdução à Demografia da Educação. Campinas: ABEP; 2004. p.45-70.

HALL, T.L.; MEJIA, J. Health Manpower Planning: principles, methods, issues. Switzerland: World Health Organization, 1978.

MAGNAGO, C. A gestão do trabalho na Estratégia Saúde da Família em municípios do Estado do Rio de Janeiro com mais de 500 mil habitantes: o caso do Rio de Janeiro e Duque de Caxias. Dissertação (Mestrado em Saúde Coletiva) - Instituto de Medicina Social, Universidade do Estado do Rio de Janeiro, Rio de Janeiro, 2012.

MAGNAGO, C. et al. The influence of family health strategy on health indicators in the municipalities of Rio de Janeiro, Brazil. Online Brazilian Journal of Nursing, v.10, n.1, 2011. Disponível em http://www.objnursing.uff.br/index.php/nursing/article/ view/j.1676-4285.2011.3211.1/html. Acesso em: 08 ago. 2011.

MARINHO, A.; CARDOSO, S.S. Um estudo multinível sobre as filas para internações relacionadas com a gravidez, o parto e o puerpério no SUS. Revista Economia Aplicada, v.11, n.4, p.527-554, 2007. Disponível em http://www.scielo.br/pdf/ecoa/v11n4/04.pdf. Acesso em: 02 set. 2011.

MARINHO, A. Um estudo sobre as filas para transplantes no Sistema Único de Saúde brasileiro. Cadernos de Saúde pública, v.22, n.10, p.2.229-2.239, out. 2006. 
MARINHO, B.L.; VASCONCELOS, E.P.G. Dimensionamento de recursos humanos: desenvolvimento de um modelo conceitual e sua aplicação. Revista de Gestão USP, v.14, n.2, p.61-76, 2007.

MATSUSHITA, M.S.; ADAMI, N.P.; CARMAGNANI, M.I.S. Dimensionamento do pessoal de enfermagem das unidades de internação do Hospital São Paulo. Acta Paulista de Enfermagem, v.18, n.1, p.9-19, 2005.

MEDEIROS, C.R.G. et al. A rotatividade de enfermeiros e médicos: um impasse na implementação da Estratégia de Saúde da Família. Revista Ciência e Saúde Coletiva, v.15, supl.1, p.1521-1531, 2010.

MENDES, K.D.S.; SILVEIRA, R.C.C.P.; GALVÃO, C.M. Revisão integrativa: método de pesquisa para a incorporação de evidências na saúde e na enfermagem. Revista Texto \& Contexto Enfermagem, v.17, n.4, p.758-64, 2008.

NEY, M.S. Condições de fixação do médico no Programa Saúde da Família Dissertação (Mestrado em Saúde da Família) - Universidade Estácio de Sá, Rio de Janeiro, 2009. 128p.

NICOLA, A.L.; ANSELMI, M.L. Dimensionamento de pessoal de enfermagem em um hospital universitário. Revista Brasileira de Enfermagem, v.58, n.2, p.186-90, 2005.

OCKÉ-REIS, C.O.; ANDREAZZI, M.F.S.; SILVEIRA, F.G. O mercado de planos de saúde no Brasil: uma criação do Estado. Revista de Economia Contemporânea, v.10, n.1, p. 157-85, 2006. Disponível em http://www.scielo.br/pdf/rec/v10n1/07.pdf. Acesso em: 20 ago. 2011.

OLIVEIRA, A.G.; BIANCHINI, D.; ABBADE, M.L.F. Métricas para dimensionar recursos humanos nos Centros de Operações de Redes [internet]. In: SIMPÓSIO BRASILEIRO DE REDES DE COMPUTADORES E SISTEMAS DISTRIBUÍDOS, 25., mai-jun 2007. Belém do Pará, 2007. Disponível em: http://www.sbrc2007.ufpa.br/ anais/2007/P01\%20-\%2004.pdf. Acesso em: 25 maio 2011.

ORGANIZAÇÃO MUNDIAL DA SAÚDE. Trabalhando juntos pela saúde. Relatório Mundial da Saúde 2006. Brasília: OPAS, 2007. 210p. Disponível em http://www.who.int/ whr/2006/06_overview_pr.pdf. Acesso em: 10 ago.2011.

PATTEN JR., T.H. Manpower Planning and the development of human resources. Canadá: John Willey \& Sons, 1971.

PICCHIAI, D. Dimensionamento quantitativo de recursos humanos em hospitais privados e públicos no Estado de São Paulo. São Paulo: Fundação Getúlio Vargas, 2000 (Relatório de pesquisa, n. 9). Disponível em: http://bibliotecadigital.fgv.br/dspace/bitstream/ handle/10438/3045/Rel09-2000.pdf?sequence=1 Acesso em: 20 maio 2011.

PIERANTONI, C.R. et al. Gestão do trabalho e da educação em saúde: recursos humanos em duas décadas do SUS. Physis: Revista de Saúde Coletiva, v.18, n.4, p.685-704, 2008. 
Planejamento de recursos humanos em saúde: desenvolvendo tecnologias para

dimensionamento de pessoal. Brasília: Ministério da Saúde, 2011.

Reconfigurando perfis profissionais: a especialização em saúde da família. In: PIERANTONI, C.R.; VIANA, A.L.D. (Org.). Educação e Saúde. São Paulo: Hucitec, 2010. p. 224-39.

RODRIGUES, F.G. Médicos em minas Gerais: projeções para o período 2010-2020 Dissertação (Mestrado em Demografia) - Faculdade de Ciências Econômicas, Universidade Federal de Minas Gerais, Belo Horizonte, 2008. 89p.

ROSA, B.A.; FILHO, E.M.S.Dimensionamento de recursos humanos em uma empresa fabricante de materiais sanitários: uma abordagem via programação inteira. In: SIMPÓSIO DE PESQUISA OPERACIONAL E LOGÍSTICA DA MARINHA, 11. 5-6 ago 2008. Rio de Janeiro, 2008. Disponível em: https://www.casnav.mar.mil.br/versoes/2009/artigos/ artigos2008/028.pdf Acesso em: 28 maio 2011.

ROSSELLI, D. et al. Estimación de la oferta de médicos especialistas en Colombia con el método de captura-recaptura. Revista Panamericana de Salud Publica, v.9, n.6, p.393-98, 2001. SOUZA, K.W. et al. Estratégias de prevenção para câncer de testículo e pênis: revisão integrativa. Revista da Escola de Enfermagem da USP, v.45, n.1, p.277-82, 2011.

TANOS, M.A.A.; MASSAROLLO, M.C.K.B.; GAIDZINSKI, R.R. Dimensionamento de pessoal de enfermagem em uma unidade especializada em transplante de fígado: comparação do real com o preconizado. Revista Escola de Enfermagem da USP, v.34, n.4, p.376-82, 2000.

VARELLA, T.C.; PIERANTONI, C.R. Mercado de trabalho: revendo conceitos e aproximando o campo da saúde. A década de 90 em destaque. Physis: Revista de Saúde Coletiva, v.18, n.3, p.521-544. 2008.

WHITTEMORE, R.; KNAFL, K. The integrative review: update methodology. Journal of Advanced Nursing, v.52, n.5, p.546-53, 2005.

\section{Nota}

${ }^{1}$ C.M.M. Vianna, C.R. Pierantoni e T. França participaram da definição do tema e dos objetivos, seleção dos artigos, construção metodológica e revisão do texto final. C. Magnago, M.P.S. Rodrigues e M.C. Morici participaram da coleta de dados, síntese e análise dos dados e concepção do manuscrito. 


\section{Abstract}

Econometric models to estimate labor force: an integrative literature review

The integrative literature review aimed to raise the current state of knowledge on econometric models of projection of the labor force in Brazil and to identify the most suitable for application in Primary Health Care. The search was conducted in several sources: electronic databases, journals, conference proceedings, crossreference and Plataforma Lattes, through descriptors and key words. The sample consisted of nine publications, which were read, summarized and analyzed. The results indicate the use of six methods to estimate labor force, but most of the methodologies propose predictive calculations for staff sizing with a view to the immediate allocation / reallocation of professionals. It emphasizes the impossibility of defining the best econometric model for the projection of health manpower in primary health care, due to the small number of national publications on the subject, which showed no homogeneity, although they display a greater concern with the allocation of health manpower, especially in hospitals.

> Key words: health manpower; labor force; econometric models; health care needs and demand; estimation techniques. 